\title{
Structure and Stability Analysis of Antibacterial Substances Produced by Selenium Enriched Bacillus Cereus
}

\section{Shanxiu Ming}

Wuhan Polytechnic University

\section{Xujun Chen}

Wuhan Polytechnic University

Na Zhang

Wuhan Polytechnic University

Shuyi Li

Wuhan Polytechnic University

Zhenzhou Zhu ( $\nabla$ zhenzhouzhu@126.com )

Wuhan Polytechnic University https://orcid.org/0000-0001-6051-5661

\section{Shuiyuan Cheng}

Wuhan Polytechnic University

\section{Research Article}

Keywords: Antibacterial Substance, Bacillus cereus, Stability, Structure analysis

Posted Date: September 22nd, 2021

DOI: https://doi.org/10.21203/rs.3.rs-888511/v1

License: (c) (i) This work is licensed under a Creative Commons Attribution 4.0 International License. Read Full License 


\section{Abstract}

Microorganisms can produce many antibiotics against bacteria and fungi, which have been used as potential choice of new antibiotics. In this paper, Bacillus cereus, was used to study the properties of antibacterial substance in its fermentation supernatant. The results showed that the source of antibacterial activity of Bacillus cereus was mainly the antibacterial substance produced by acid precipitation method, and further characterization speculates that the antibacterial substance may be lipopeptide substance. Then the antibacterial spectrum of the antibacterial substance was investigated, which showed that the antibacterial substance only had good inhibitory effect on Gram-positive bacteria and fungi, and selenium enrichment could significantly enhance the antibacterial activity of lipopeptide antibacterial substance produced by Bacillus cereus, and the inhibition mode of antibacterial substance to indicator bacteria was determined. The effects of different treatment methods on the stability of antibacterial substance were studied and the results showed that the antibacterial substance were stable to heat, ultrasonic and ultraviolet treatment, and their antibacterial activity would not be greatly affected. However, they were sensitive to pepsin. The optimum $\mathrm{pH}$ range of antibacterial activity was 3-5. This study may contribute to reuse the fermentation supernatant often discarded in the previous fermentation process. At the same time, the lipopeptide antibacterial substance extracted from the fermentation broth of selenium enriched Bacillus cereus can be used in the development of antibiotics and biopesticides, and open up a new way for the control of plant diseases.

\section{Introduction}

Since the discovery of penicillin in 1928, antibiotics have been considered as the only effective way to inhibit microorganisms. But the researchers still have not given up the search for new and more effective antibiotics (Guy and Mallampalli, 2008). At present, although the research of antibiotics has made a great breakthrough, the use of antibiotics has been close to saturation. Due to the shortcomings of some antibiotics and the inappropriate use of antibiotics by manufacturers, microbial resistance to antibiotics has been enhanced, thus limiting the use of antibiotics (Blomberg, 2008). Based on the above facts, while reducing microbial resistance to antibiotics, the demand for new antibiotics is also increasing, and natural antibiotics are the potential choice of new antibiotics. Microorganisms can produce many natural antibacterial substance to inhibit bacteria and fungi, and are also an important source of antibiotics for various diseases (Thakur et al., 2007). Therefore, the production of natural antibiotics by microorganisms is of great significance.

Members of bacillus are facultative anaerobic or oxygen aerobic Gram-positive bacteria that can produce a variety of lipopeptide antibiotics (Liang et al., 2017), regulate the intestinal microecological balance, promote intestinal development and nutrient absorption, and is one of the widely used strains (Du et al., 2017). At present, it has been known that Bacillus subtilis, Bacillus cereus, Bacillus amyloliquefaciens, Bacillus licheniformis, Bacillus pumilus, Bacillus thuringiensis, Bacillus circulans and Bacillus brevis can produce a variety of antimicrobial substance including lipopeptides, peptides and bacteriocins (Gordon et al., 1983). Bacillus cereus is a facultative anaerobic Gram-positive bacillus, which widely exists in soil 
and other environments, it can secrete a variety of secondary metabolites against bacterial and fungal, including lipopeptide antibiotics synthesized by non ribosomal peptide synthetase (NRPS). Antimicrobial substance produced by microorganisms have the advantages of high safety, less side effects and less drug resistance compared with chemical synthetic antibiotics (Fengying Li et al., 2016a).

Previous studies have shown that inorganic selenium can be converted into organic selenium to produce selenium enriched strains by using microbial strains (Wang and Fang, 2011). Many strains have the effect of inhibiting intestinal harmful bacteria, and many studies have shown that this effect can be significantly improved after selenium enrichment. On the one hand, selenium can reduce the microbial activity, on the other hand, it can improve the antibacterial properties of the fermentation products. Generally speaking, the antibacterial activity of selenium enriched strains is affected by the corresponding strain carriers. At present, the research on selenium enriched strains is more about lactic acid bacteria and yeast, but less about Bacillus cereus. Lipopeptide antibacterial substance is one of the main antibacterial substance produced by Bacillus, which has been considered to be an important reason for its ability to inhibit plant diseases as a biological pesticide (Hsieh et al., 2008). It is a cyclic peptide composed of fatty acids and peptide chains, and has good preventive effect on Aspergillus flavus (Moyne et al., 2001), scab (Xiang et al., 2015), Fusarium wilt (Xiang et al., 2016) and many plant diseases. Zhang and others optimized the culture medium and fermentation conditions of a Bacillus subtilis strain producing lipopeptide antibacterial substance, and successfully increased the antibacterial potency of the antibacterial substance by 3.51 times, and achieved the effect of high yield, low cost and short fermentation time (Zhiyan Zhang et al., 2019). Jiang et al. studied the lipopeptide antibacterial substance produced by rapeseed endophytic bacterium and the results showed that the extracellular secretory substance contained peptide bond and had ring structure, then the antibacterial substance was identified as surfactin of cycle lipopeptide antibiotics by high performance liquid chromatography and mass spectrometry (Jiang et al., 2010).

The purpose of this study was to investigate the properties of antibacterial substance in the fermentation supernatant of Bacillus cereus. The antibacterial substance of protein and lipopeptide were extracted from the fermentation supernatant of Bacillus cereus by ammonium sulfate precipitation method and acid precipitation method respectively, and their antibacterial activities were compared to determine the main source of antibacterial activity of Bacillus cereus. The antibacterial substance was preliminarily analyzed by biuret method and ninhydrin method, and further analyzed by Fourier transform infrared spectroscopy, nuclear magnetic resonance spectroscopy and matrix assisted laser desorption time of flight mass spectrometry. And then the action mode of antibacterial substance on indicator bacteria was studied, and the effects of different treatment methods on the stability of antibacterial substance were compared.

\section{Material And Methods Strain material}


The experimental Bacillus cereus strain were isolated from soil that was taken from Enshi (China) and obtained by Se-Run Health Tech Development Co., Ltd in Enshi (China). The strain was collected at $-80^{\circ} \mathrm{C}$ and inoculated into Luria-Bertani (LB) agar medium plates, activated at $37^{\circ} \mathrm{C}$ for $6 \mathrm{~h}$, and then single colonies were selected from it and inoculated into liquid LB medium. The strain seed liquid was obtained by culture at 150rpm on a shaking incubator HZ-150L(Ruihua Instrument \& Equipment, China) with a rotational radius of $20 \mathrm{~mm}$ (same rotational radius below) for $12 \mathrm{~h}$, and the concentration is $3 \times 10^{7} \mathrm{cfu} / \mathrm{mL}$.

\section{Chemicals and reagents}

Analytical grade sodium selenite, analytical grade hydrochloric acid, sodium chloride, copper sulphate, ammonium sulphate, methanol, and ninhydrin were purchased from Sinopharm Chemical Reagent Co.,Ltd (Shanghai, China). Guaranteed potassium bromide was purchased from Aladdin Biochemical Technology Co.,Ltd(Shanghai, China). Tryptone was purchased from OXOID (Hants, UK). Yeast Extract was purchased from Aoboxing Biotechnology (Beijing, China). Agar was purchased from Biofroxx (Germany). Pepsin was purchased from Solarbio (Beijing, China).

\section{Isolation and characterization of antimicrobial substance from Bacillus cereus supernatant}

\section{Identification of the source of bacteriostasis in the supernatant of Bacillus cereus}

The antibacterial activity of antibacterial substance solution produced by Bacillus cereus was determined by Oxford cup method (Zhengyu Fan et al., 2018). The strain seed liquid was inoculated into $2 \mathrm{~L}$ liquid LB medium with $7 \%(\mathrm{v} / \mathrm{v})$ inoculation amount, and selenium enriched group and non selenium enriched group were set up. The selenium enriched group was cultivated at $33^{\circ} \mathrm{C}$ and $170 \mathrm{rpm}$ for $4 \mathrm{~h}$, and then added with $10 \mathrm{mg} / \mathrm{L}$ sodium selenite solution to make the concentration of sodium selenite reach $150 \mu \mathrm{g} / \mathrm{mL}$, and then continue to cultivate for $24 \mathrm{~h}$. The non selenium enriched group was cultivated at $33^{\circ} \mathrm{C}$ and $170 \mathrm{rpm}$ for $24 \mathrm{~h}$. Then the bacterium solution was centrifuged at $4000 \mathrm{rpm}$ for $25 \mathrm{~min}$ to collect the supernatant, and then sterilized by $0.22 \mu \mathrm{m}$ membrane. Finally, the supernatant was concentrated 10 times by rotary evaporator to obtain the supernatant containing antibacterial substance of Bacillus cereus, and then the antibacterial substance were prepared by ammonium sulfate precipitation and acid precipitation respectively. The detailed methods are as follows (Hongxiao Li et al., 2016b):

1. Extraction of antimicrobial substance from Bacillus cereus by ammonium sulfate precipitation 
$62.6 \mathrm{~g}$ ammonium sulfate was ground into powder, and then added into $200 \mathrm{~mL}$ supernatant containing antibacterial substance at room temperature while stirring, so that the ammonium sulfate saturation in the solution was $50 \%$, then the mixed solution was placed at room temperature for $6 \mathrm{~h}$ to precipitate the antibacterial substance. After that, the solution was centrifuged at $4{ }^{\circ} \mathrm{C}$ and $6000 \mathrm{rpm}$ for $30 \mathrm{~min}$. The precipitates were freeze-dried and stored for use.

2. Extraction of antimicrobial substance from Bacillus cereus by acid precipitation

The supernatant containing antibacterial substance was adjusted to $\mathrm{pH} 2.0$ with $6 \mathrm{~mol} / \mathrm{L} \mathrm{HCl}$ and precipitated overnight at $4{ }^{\circ} \mathrm{C}$, and then centrifuged at $4{ }^{\circ} \mathrm{C}$ for $30 \mathrm{~min}$ at $6000 \mathrm{rpm}$. After discarding the supernatant, methanol was added into the precipitate and stirring for $2 \mathrm{~h}$, then the solution was centrifuged at $4{ }^{\circ} \mathrm{C}$ and $6000 \mathrm{rpm}$ for $20 \mathrm{~min}$, and the methanol solution at the upper layer was collected. Adding $1 \mathrm{~mol} / \mathrm{L} \mathrm{NaOH}$ to adjust the $\mathrm{pH}$ to 7.0 , then rotating evaporation at $45^{\circ} \mathrm{C}$ to remove methanol, freeze-drying the remaining aqueous solution to obtain antibacterial material powder for use.

The antibacterial activity of the two antibacterial substance solution was compared with the initial supernatant to explore and separate the antibacterial substance which play a major role in the supernatant.

\section{Preliminary analysis of antimicrobial substance produced by Bacillus cereus}

The antibacterial substance was preliminarily inferred by biuret method and ninhydrin method, and the detailed methods are as follows (Chen, 2008):

1. Biuret reaction of antibacterial substance

The antibacterial substance solution and control solution were added to the two test tubes respectively. Add 10 drops of $10 \%$ sodium hydroxide and 2 drops of $1 \%$ copper sulfate into each test tube, and observe the color change of the solution after full mixing.

2. Ninhydrin reaction of antibacterial substance

Two tubes were added with antibacterial solution, another tube was added with control solution, and then ninhydrin reagent was added to each tube and the color change of solution was observed. Then put a tube of antibacterial solution into a boiling water bath and observe the color change of the solution again.

\section{Analysis of antimicrobial substance produced by Bacillus cereus}

\section{Infrared spectrum analysis of antibacterial substance}


Appropriate amount of antibacterial substance samples were mixed with potassium bromide powder evenly at room temperature, and then the mixed powder was extruded into flakes through a tablet press. The sample pieces were put into an infrared spectrometer Nicolet NEXUS670(Thermo Nicolet Corporation, US) for infrared spectrum scanning, with the scanning range of $4000-400 \mathrm{~cm}^{-1}$.

\section{Analysis of antibacterial substance by time of flight mass spectrometry}

The molecular weight of lipopeptide antimicrobial substance produced by Bacillus is generally in the range of $900-2000$ (Youzhou Liu et al., 2017). The sample plate was dripped with $1 \mu \mathrm{L}$ methanol solution of antibacterial substance and covered with $1 \mu \mathrm{L}$ a-Cyano-4-hydroxycinnamic acid matrix working solution. After the plate was naturally dried, time of flight mass spectrometry AB SCIEX MALDITOF/TOF 5800(AB Sciex Pte. Ltd., US) was used for high-resolution positive ion detection under $337 \mathrm{~nm}$ excitation light source.

\section{NMR analysis of antimicrobial substance}

$20 \mathrm{mg}$ antibacterial substance sample was accurately weighed and dissolved in deuterated methanol, and determined by nuclear magnetic resonance BRUKER AVANCE 400(Bruker Corporation, Germany) with 16 scanning times.

\section{Determination of antibacterial spectrum}

Staphylococcus aureus, Micrococcus gambiae, Escherichia coli, Salmonella, yeast and Aspergillus niger were inoculated into LB liquid medium respectively until the colony total was approximately the same, and then $100 \mu \mathrm{L}$ bacterial liquid was spread on LB solid medium, and Oxford cup was placed on the medium, the antibacterial substance solution obtained from selenium enriched and non selenium enriched culture was added into the Oxford cup respectively. Then the diameter of inhibition zone was determined after $24 \mathrm{~h}$ incubation at $37^{\circ} \mathrm{C}$ to obtain the antibacterial spectrum of Bacillus cereus.

\section{Study on the antibacterial action mode of antibacterial substance produced by Bacillus cereus}

A small agar block was cut from the bacteriostatic area of the plate with inhibition zone, and put it into 10 $\mathrm{mL}$ liquid LB medium. Then the agar block was broken into pieces by Vortex oscillator(IKA, Germany) and was cultivated at $37^{\circ} \mathrm{C}$ and $150 \mathrm{rpm}$ for $24 \mathrm{~h}$. The initial and final $\mathrm{OD}_{600 \mathrm{~nm}}$ of bacterial liquid were determined, and the viable bacteria were counted (Hao et al., 2006). 
Add $10 \mathrm{~mL}$ LB liquid medium into two tubes respectively, and add bacteriostatic substance solution into one tube and control solution into the other tube. After that, the activated indicator bacteria solution was inoculated into it and cultured at $37^{\circ} \mathrm{C}$ for $3 \mathrm{~h}$, then the supernatant was discarded and the precipitates of two groups of bacteria were collected after centrifugation. The precipitates of two groups of bacteria were collected and washed repeatedly with sterile water before freeze-drying. The two groups of bacteria powder were placed under scanning electron microscope for microscopic morphology observation.

\section{Stability analysis of antibacterial substance produced by Bacillus cereus}

$1 \mathrm{~mL}$ of antibacterial substance solution produced by Bacillus cereus cultured in selenium enriched and non selenium enriched medium were respectively put into test tube, then treated at $40{ }^{\circ} \mathrm{C}, 60{ }^{\circ} \mathrm{C}, 80^{\circ} \mathrm{C}$ and $100{ }^{\circ} \mathrm{C}$ for $30 \mathrm{~min}$ for thermal stability analysis, and $20,30,40$, and $50 \mathrm{~min}$ with ultrasonic (100 W) treatment for ultrasonic stability, the ultraviolet stability was analyzed by exposure the antibacterial substance solution to ultraviolet lamp (40 W) for 20,30, 40 and $50 \mathrm{~min}$, and the $\mathrm{pH}$ value of antibacterial substance solution was adjusted to $3,5,7,9$, and 11 with $0.1 \mathrm{~mol} / \mathrm{L} \mathrm{NaOH}$ or $0.1 \mathrm{~mol} / \mathrm{L} \mathrm{HCl}$, then the $\mathrm{pH}$ value was returned to the neutral after $24 \mathrm{~h}$ of treatment at $37^{\circ} \mathrm{C}$ for acidic and alkaline stability analysis. The stability of antibacterial substance solution under the action of pepsin was analyzed by adding 1 $\mathrm{mg} / \mathrm{mL}$ pepsin to the supernatant at $37^{\circ} \mathrm{C}$ and $\mathrm{pH} 7.0$ for $6 \mathrm{~h}$.

$100 \mu \mathrm{L}$ of indicator bacteria liquid was spread on LB solid medium, and aseptic Oxford cup was placed in medium. The antibacterial substance solution produced by Bacillus cereus cultured in selenium enriched and non selenium enriched medium were added to Oxford cup respectively, and the control solution was set. The change of antibacterial activity was determined by measuring the diameter of inhibition zone.

\section{Statistical analysis}

All trials were carried out in triplicate and all the data were reported as means $\pm S D$ (standard deviation). The statistics significance was evaluated using Student's t-test and $\mathrm{P}<0.05$ or 0.01 was taken as significant.

\section{Results And Discussion}

\section{Identification of the source of bacteriostasis in the supernatant of Bacillus cereus}

As shown in Figure 1, compared with the control of the supernatant containing antibacterial substance of Bacillus cereus, the antibacterial substance obtained by acid precipitation method have almost the same antibacterial activity, while the antibacterial substance obtained by ammonium sulfate precipitation method do not show obvious antibacterial effect. Therefore, it is inferred that the antibacterial activity of 


\section{Preliminary analysis of antimicrobial substance produced by Bacillus cereus}

1. Biuret reaction of antibacterial substance

It can be seen from Figure 2(a,b) that biuret reaction of antibacterial substance produced by Bacillus cereus showed positive of purple phenomenon. This indicates that antibacterial substance produced by Bacillus cereus contains peptide bond, which may belong to protein or polypeptide.

2. Ninhydrin reaction of antibacterial substance

It can be seen from Figure 2(c,d,e) that there was no color change when ninhydrin was added to the aqueous solution of antibacterial substance produced by Bacillus cereus, so it is inferred that the antibacterial substance does not contain free a-amino group. After boiling in boiling water bath for a few minutes, the solution in the tube appeared positive reaction and turned purple, which indicated that heating promoted the partial decomposition of antibacterial substance and produced free a-amino group.

In conclusion, the structure of antibacterial substance produced by Bacillus cereus has peptide bond, and does not contain free a-amino group. Therefore, it is preliminarily inferred that the antibacterial substance belongs to the cyclic peptide. Cheng et al. (2009) isolated and analyzed the antimicrobial peptide produced by the endophytic bacteria PCE45 of Paris polyphylla var. chinensis. The results showed that the ninhydrin reaction test was negative, indicating that the substance had no free $\mathrm{N}$-terminal. After acid hydrolysis, the ninhydrin reaction was positive again, so it was speculated that the substance might be a cyclic peptide. Lanyan Fan et al. (2013) carried out color reaction test on czk1 antibacterial substance produced by Endophytic Bacillus subtilis from Hevea brasiliensis. The results showed that the antibacterial substance solution had obvious color change after biuret reaction for $10 \mathrm{~min}$. There was no obvious reaction after mixing with ninhydrin, and the color became darker after boiling water heating, indicating that the substance may belong to cyclic peptide.

\section{Structure analysis of antibacterial substance produced by Bacillus cereus}

\section{Infrared spectrum analysis of antibacterial substance}

As shown in Figure 3, the characteristic absorption peak at $3220.71 \mathrm{~cm}^{-1}$ is caused by the stretching vibration of amide bond, indicating the existence of peptide bond, and the characteristic absorption at $1733.63 \mathrm{~cm}^{-1}$ is caused by the contraction vibration of lactone carbonyl, it shows that there is a structure 
of $\mathrm{C}=\mathrm{O}$ of ester carbonyl, and $1630.75-1659.10 \mathrm{~cm}^{-1}$ is the stretching vibration of free amino group and ketone group, indicating the existence of $\mathrm{N}-\mathrm{H}$ group and $\mathrm{C}=\mathrm{O}$ group. The above characteristic absorption indicates that the antibacterial substance has peptide chain. The absorption at $1402.41 \mathrm{~cm}^{-1}$ and $1453.24 \mathrm{~cm}^{-1}$ is the stretching vibration of $\mathrm{C}-\mathrm{H}$ bond in aliphatic carbon chain, and the stretching vibration of aromatic $=\mathrm{C}-\mathrm{H}$ group at $3055.17 \mathrm{~cm}^{-1}$. The above characteristic absorption indicates that the antibacterial substance contains fatty acid molecules. $1093.19 \mathrm{~cm}^{-1}$ is the stretching vibration of C-O-C bond. Based on the above results, it is inferred that the antibacterial substance produced by Bacillus cereus is a kind of lipopeptide with ring structure. Similar results were found in the spectral properties of lipopeptide surfactants produced by Alkalihalobacillus clausii (Hazra et al., 2014), Bacillus pumilus (Morikawa et al., 1992) and Bacillus licheniformis (Yakimov et al., 1995).

\section{Analysis of antibacterial substance by time of flight mass spectrometry}

As shown in Figure $4(A)$, there are ion peaks at $\mathrm{m} / \mathrm{z} 1008.502, \mathrm{~m} / \mathrm{z} 1022.536$ and $\mathrm{m} / \mathrm{z} 1036.588$, and the molecular weight difference is $14 \mathrm{Da}$, which was exactly the length of a fatty acid chain $\left(-\mathrm{CH}_{2}\right)$. It was speculated that these ion peaks are the molecular weight of a group of surfactin which are homologous to each other (C13-C15). As shown in Figure 4(B), there are ion peaks at $\mathrm{m} / \mathrm{z} 1021.533, \mathrm{~m} / \mathrm{z} 1035.533$ and $\mathrm{m} / \mathrm{z} 1049.577$, with a molecular weight difference of $14 \mathrm{Da}$. It was speculated that these ion peaks are the molecular weights of a group of bacillomycin L (C14-C16) which are homologous to each other. The above results were consistent with the reports in the literature (Luo et al., 2015).

\section{NMR analysis of antimicrobial substance}

As shown in Figure 5, multiple overlapping methylene $\left(-\left(\mathrm{CH}_{2}\right)_{\mathrm{n}^{-}}\right)$signal characteristic resonances were observed in the range of 1.45-1.0 ppm, indicating the existence of long-chain fatty acid structure in the molecule. The resonance signal between 5.2 and $4.4 \mathrm{ppm}$ indicates the presence of fatty acid functional groups and $\mathrm{C}-\mathrm{H}$ bonds in peptide. The characteristic signal in the range of 8.0-7.2ppm indicates the existence of $\mathrm{N}-\mathrm{H}$ characteristic resonance in peptide chain. At the same time, the figure also shows the presence of $-\mathrm{C}-\mathrm{OH}$ structure( $(2.17 \mathrm{ppm})$, and the signal at the chemical shift of $3.37 \mathrm{ppm}$ was the solvent peak produced by deuterated methanol. Based on the above results, it was speculated that there is lipopeptide structure in the antibacterial substance (Sharafi et al., 2014). Andreia et al. studied the biosurfactant produced by a Bacillus subtilis strain Isfm-0 5 by ${ }^{1} \mathrm{H}$ NMR. The results clearly showed that the substance had long fatty acid chain, peptide main chain and aliphatic carbon hydrogen bond, indicating that the surfactant was lipopeptide (Faria et al., 2011). Liu et al. also reported the same results. They confirmed and characterized c15-surfactant-o-methyl ester produced by Bacillus subtilis HSO 121, a lipopeptide producing strain (Xiangyang Liu et al., 2009). 


\section{Determination of antibacterial spectrum}

After the indicator bacteria were cultured for 24 hours, a clear antibacterial circle appeared around the Oxford cup on the plates of Staphylococcus aureus, Micrococcus luteus and Aspergillus niger, but there was no significant change on the plates of Salmonella, Escherichia coli and yeast. The measured diameter of inhibition zone was shown in Table 1. It can be seen that the antibacterial substance produced by Bacillus cereus has inhibitory effect on Gram-positive bacteria and some fungi, but has no inhibitory effect on Gram-negative bacteria, and selenium enrichment can significantly enhance its antibacterial activity. Oscáriz et al. studied the bacteriocin cerein 7 produced by a Bacillus cereus in 1999 and 2000, and the results showed that this antibacterial substance had broad-spectrum antibacterial activity against Gram-positive bacteria, but had no inhibitory effect on Gram-negative bacteria (Oscáriz et al., 1999; Oscáriz and Pisabarro, 2010). In 2009, Lappe and others found that cerein 8A, an antibacterial substance produced by Bacillus cereus, could inhibit Gram-negative bacteria including Salmonella (Lappe et al., 2009). It can be seen that the range of antibacterial spectrum of the strains in this study is different from that of other similar studies, which may be due to the great differences in DNA level and physicochemical properties of different Bacillus cereus, resulting in the differences in physicochemical properties, antibacterial mechanism and chemical structure of antibacterial substance produced by the strains (Lina Zhang, 2012). At the same time, due to the obvious inhibitory effect of the antibacterial substance on Staphylococcus aureus, Staphylococcus aureus was selected as the indicator bacteria in the follow-up test.

Table 1. Inhibition spectrum of Bacillus cereus.

\begin{tabular}{|llll|}
\hline & Indicator bacteria & \multicolumn{2}{l|}{ Diameter of bacteriostatic zone/mm } \\
\cline { 2 - 4 } & $\begin{array}{l}\text { Selenium } \\
\text { enrichment }\end{array}$ & $\begin{array}{c}\text { Non Selenium } \\
\text { enrichment }\end{array}$ \\
\hline Gram positive bacteria & $\begin{array}{l}\text { Staphylococcus } \\
\text { aureus }\end{array}$ & $15.1 \pm 0.2$ & $9.0 \pm 0.2$ \\
\cline { 2 - 4 } $\begin{array}{l}\text { Micrococcus luteus } \\
\text { Gacteria negative }\end{array}$ & $11.2 \pm 0.6$ & $8.7 \pm 0.4$ \\
\hline Fungus & Escherichia coli & - & - \\
\hline Salmonella & - & - \\
\hline & Saccharomycetes & - & - \\
\hline & Aspergillus niger & $13.2 \pm 0.4$ & $8.7 \pm 0.2$ \\
\hline
\end{tabular}

\section{Morphological effects of antibacterial substance produced by Bacillus cereus on indicator bacteria}


As shown in Table 2, the number of living bacteria in the medium increased significantly after the agar block in the inhibition zone was cultured at $37^{\circ} \mathrm{C}$ for $24 \mathrm{~h}$, indicating that the indicator bacteria in the inhibition zone were not completely killed by the antibacterial substance, and its inhibition mode was to temporarily inhibit the growth activity of the indicator bacteria, and the indicator bacteria could continue to grow when the conditions were appropriate (Lina Zhang, 2012).

The effect of antibacterial substance on Staphylococcus aureus was observed by scanning electron microscope. The results are shown in Figure 6. In the blank control group, Staphylococcus aureus showed the characteristics of full shape, smooth and complete surface, clear outline, and obvious spherical shape. However, after treated with antibacterial substance produced by Bacillus cereus for 3 hours, the surface of Staphylococcus aureus in the experimental group became rough, and a large number of cells showed folds and depressions with abnormal cell morphology. This indicates that the antibacterial substance can effectively destroy the surface structure of the cells and make their morphology abnormal. Cao et al. (2019) studied the antibacterial mechanism of Bacillus subtilis fermentation extract on E.coli by scanning electron microscope. The results showed that the surface of E.colicells became rough and wrinkled after the effect of the extract, and a large number of abnormal morphological bacteria appeared, which confirmed the destructive effect of antibacterial substance produced by Bacillus subtilis on the surface structure of indicator cells.

Table 2. The $\mathrm{OD}_{600 \mathrm{~nm}}$ and bacterial accounts antibacterial circle area.

\begin{tabular}{|lll|}
\hline & Oh & $24 \mathrm{~h}$ \\
\hline $\mathrm{OD}_{600 \mathrm{~nm}}$ & 0.122 & 1.7045 \\
\hline Number of live bacteria /cfu & $1.6 \times 10^{3}$ & $2.3 \times 10^{9}$ \\
\hline
\end{tabular}

\section{Stability analysis of antibacterial substance produced by Bacillus cereus}

As shown in Figure 7, selenium enrichment can significantly enhance the antibacterial activity of antibacterial substance produced by Bacillus cereus. It seems that selenium promotes the production of soluble metabolites related to antibacterial activity. When selenium replaces sulfur in disulfide bond of antibacterial peptide, its antibacterial activity may be improved. Of course, the increase of biomass may also be the reason for the enhancement of antibacterial activity. Kheradmand et al. (2014) also found a similar phenomenon in the experiment, the antibacterial activity of Lactobacillus plantarum and Lactobacillus johnsonii against Candida albicans was increased after selenium enrichment.

In addition, after different levels of temperature, ultrasound and UV treatment, the antibacterial activity of antibacterial substance decreased slightly, which may be due to the partial inactivation of antibacterial substance caused by high temperature, high power ultrasonic and ultraviolet treatment. But in general, 
the antibacterial substance produced by Bacillus cereus had good stability to temperature, ultrasound and UV treatment $(\mathrm{p}<0.05)$. At the same time, the antibacterial substance have antibacterial activity in the range of $\mathrm{pH} 3-11$, but the optimal $\mathrm{pH}$ range for its antibacterial activity is $\mathrm{pH} 5-7$. The $\mathrm{pH}$ value above or below this range will lead to a significant decrease in antibacterial activity. P A et al. (2010) isolated a substance with antibacterial activity from Bacillus cereus ATCC 14579 in 2004. It has inhibitory effect on clinically related Staphylococcus aureus and Micrococcus /uteus, and has high antibacterial activity in the range of $\mathrm{pH}$ 2.0-9.0. And after pepsin treatment, the antibacterial activity of the antibacterial substance solution decreased significantly, which confirmed that the antibacterial substance was protein or polypeptide again.

\section{Conclusion}

In this study, the antibacterial substance were extracted from the fermentation broth of Bacillus cereus by ammonium sulfate precipitation method and acid precipitation method respectively, and the inhibition zone test proved that the antibacterial activity of Bacillus cereus mainly came from the substance extracted by the latter method. The results of biuret reaction and ninhydrin reaction showed that the antibacterial substance belonged to protein or peptide. Furthermore, the results of FTIR, NMR and MALDITOF-MS indicated that the antibacterial substance might be lipopeptide. The results of antibacterial spectrum test showed that Bacillus cereus had good inhibitory effect on Gram-positive bacteria and fungi, but no obvious inhibitory effect on Gram-negative bacteria, and selenium enrichment could significantly enhance the antibacterial activity of lipopeptide antibacterial substance produced by Bacillus cereus. And the mechanism of the antibacterial substance on the indicator bacteria is inhibition rather than killing, so when the growth conditions are suitable, the inhibited indicator bacteria can grow again, and the antibacterial substance can effectively destroy the surface structure of the cell, making its morphology appear abnormal changes. The results of stability test showed that the antibacterial substance produced by Bacillus cereus were stable to heat treatment, ultrasonic time treatment and ultraviolet treatment. In the range of $\mathrm{pH} 3-11$, the antibacterial substance has antibacterial activity, but the optimum $\mathrm{pH}$ range is 3-5. The antimicrobial substance produced by Bacillus cereus was sensitive to pepsin, which confirmed that the antimicrobial substance was peptide substance again. The antibacterial substance showed good stability, which provided theoretical foundation for their structural identifications and future applications. This study may contribute to provide a theoretical basis and idea for the extraction and reuse of metabolites such as antibacterial substance in the fermentation broth often discarded in the previous fermentation process. Subsequently, the antibacterial substance can be studied by means of genetic engineering, and try to construct and express it in other organisms. At the same time, this study confirmed that selenium enrichment can improve the antibacterial activity of the strain, which is conducive to the further utilization of selenium enriched Bacillus cereus. However, the mechanism of selenium enrichment enhancing antibacterial activity needs further study.

\section{Declarations}




\section{Funding}

The research was funded by Major Technology Innovation of Hubei Province (2019ABA113), the National youth talent support program in food industry of China, the Scientific and Technology Project of Enshi Tujia\&Miao Autonomous Prefecture (D20180004), and the program of the Minister of Science and Higher Education entitled "Regional Initiative of Excellence" in 2019-2022 (project no. 026/RID/2018/19).

\section{Conflicts of interest}

The authors declare no conflict of interest.

\section{Availability of data and material}

Samples of the strain are not available from the authors.

\section{Code availability}

Not applicable.

\section{Authors' contributions}

Conceptualization: Zhenzhou zhu; Methodology: Shanxiu Ming, Xujun Chen and Shuyi Li; Formal analysis and investigation: Shanxiu Ming, Xujun Chen; Data curation: Shanxiu Ming; Writing-original draft preparation: Xujun Chen, Na Zhang; Writing-review and editing: Shanxiu Ming, Xujun Chen and Zhenzhou zhu; Funding acquisition: Shuiyuan Cheng; Resources: Zhenzhou zhu; Supervision: Shuyi Li and Zhenzhou zhu; Project administration: Shuiyuan Cheng.

\section{Research involving Human Participants and/or Animals}

Not applicable.

\section{Informed consent}

Not applicable.

\section{References}

Blomberg, B. (2008). Antimicrobial resistance in developing countries. Norwegian Medical Association, 128(21), 2462.

Cao, S., Nan, N., Yuan, Y., \& Qi, X. (2019). Inhibitory Effect of Fermented Extracts by Bacillus subtilis on Escherichia coli. Science and Technology of Food Industry, 40(3), 111-115,126.

Chen, J. (2008). Biochemistry experiment. 4th Edition. The Science Publishing Company. 
Cheng, Y., Yong, B., Zhang, C., \& Liu, Q. (2009). Purification and characterization of an antimicrobial peptide from Paris polyphylla var. chinensis. Acta Microbiologica Sinica, 49(4), 498-503.

Du, J., Zhang, A., Hao, J., Zhang, X., Yang, B., Si, X., et al. (2017). Antimicrobial Activity of a BlendBiosurfactant of Lipopeptide and Glycolipid Produced by Bacillus sp. dhs-330. Letters in Biotechnology, 28(6), 818-822.

Fan, L., He, C., Liang, Y., Zheng, X., Wu, W., \& Li, R. (2013). Characteristics of the Antimicrobial Active Component of Bacillus subtilis Strain Czk1. Journal of South China University of Tropical Agriculture, $4(1), 36-44,55$.

Fan, Z., Hu, L., Sun, H., Tang, W., Gao, Z., Cui, J., et al. (2018). Screening, identifing and antimicrobial substance stability analysis of lactic acid bacterium FY-1. Food Science and Technology, 43(5), 15-18.

Faria, A. F., StéfaniTeodoro-Martinez, D., Barbosa, G. N. O., Vaz, B., Silvaa, Í., SimoneGarciab, J., et al. (2011). Production and structural characterization of surfactin (C 14/Leu 7) produced by Bacillus subtilis isolate LSFM-05 grown on raw glycerol from the biodiesel industry. Process Biochemistry, 46(10), 19511957.

Gordon, R. E., Haynes, W. C., \& Pang, C. H.-N. (1983). The Genus Bacillus. Agricultural Research Service, United States Department of Agriculture.

Guy, E. S., \& Mallampalli, A. (2008). Managing TB in the 21 st century: existing and novel drug therapies. Therapeutic Advances in Respiratory Disease, 2(6), 401-408.

Hao, H., Jia, J., Han, J., \& Niu, T. (2006). Identification of Antagonistic Bacillus sp. R21 -4 and Research of Its Antimicrobial Mechanism. Food and Fermentation Industries, 32(10), 54-58.

Hazra, C., Kundu, D., \& Chaudhari, A. B. (2014). Lipopeptide biosurfactant from Bacillus clausii BS02 using sunflower oil soapstock: evaluation of high throughput screening methods, production, purification, characterization and its insecticidal activity. RSC Advances, 5(4), 2974-2982.

Hsieh, F., Lin, T., Meng, M., \& Kao, S. (2008). Comparing Methods for Identifying Bacillus Strains Capable of Producing the Antifungal Lipopeptide Iturin A. Current Microbiology, 56(1), 1-5.

Jiang, M., Wang, G., Hu, X., Zhang, Y., \& Wan, X. (2010). Antibiotic molecular structure identification of endophytic bacterial strain BY-2. Cinses Journal of Oil Crop Sciences, 32(2), 279-284.

Kheradmand, E., Rafii, F., Yazdi, M. H., Sepahi, A. A., Shahverdi, A. R., \& Oveisi, M. R. (2014). The antimicrobial effects of selenium nanoparticle-enriched probiotics and their fermented broth against Candida albicans. DARU Journal of Pharmaceutical Sciences, 22(1), 1-6.

Lappe, R., S.Motta, A., VoltaireSant'Anna, \& Brandelli, A. (2009). Inhibition of Salmonella Enteritidis by cerein 8A, EDTA and sodium lactate. International Journal of Food Microbiology, 135(3), 312-316. 
Li, F., Wang, Y., Ma, F., \& Xue, P. (2016a). Research progress of natural antibiotics. Journal of North Pharmacy, 13(12), 119-121.

Li, H., Zhang, D., Zhao, H., Liu, W., \& Lu, C. (2016b). Physical and Chemical Properties of Antimicrobial from Bacillus amyloliquefaciens Strain MH71 and Its Inhibition Activities against Botrytis cinerea. Chinese Journal of Biological Control, 32(4), 485-492.

Liang, Y., Huang, X., Wu, W., Li, R., Zheng, J., Xi, J., et al. (2017). Optimizing Fermentation Condition for Bacillus amyloliquefaciens TWC2. Sugar Crops of China, 39(6), 17-22.

Liu, X., Yang, S., \& Mu, B. (2009). Production and characterization of a C15-surfactin-0-methyl ester by a lipopeptide producing strain Bacillus subtilis HS0121. Process Biochemistry, 44(10), 1144-1151.

Liu, Y., Chen, X., Liang, X., Qian, Y., Qiao, J., \& Liu, Y. (2017). Identification of Bacillus atrophaeus YL3 and Detection of Its Lipopeptide Compounds. Chinese Journal of Biological Control, 33(1), 142-150.

Luo, C., Zhou, H., Zou, J., Wang, X., Zhang, R., Xiang, Y., et al. (2015). Bacillomycin L and surfactin contribute synergistically to the phenotypic features of Bacillus subtilis 916 and the biocontrol of rice sheath blight induced by Rhizoctonia solani. Applied Microbiology and Biotechnology, 99(4), 1897-1910.

Morikawa, M., Ito, M., \& Imanaka, T. (1992). Isolation of a new surfactin producer Bacillus pumilus A-1, and cloning and nucleotide sequence of the regulator gene, psf-1. Journal of Fermentation and Bioengineering, 74(5), 255-261.

Moyne, A. L., Shelby, R., Cleveland, T. E., \& Tuzun, S. (2001). Bacillomycin D: an iturin with antifungal activity against Aspergillus flavus. Journal of Applied Microbiology, 90(4), 622-629.

Oscáriz, J. C., Lasa, I., \& Pisabarro, A. G. (1999). Detection and characterization of cerein 7, a new bacteriocin produced by Bacillus cereus with a broad spectrum of activity. FEMS microbiology letters, 178(2), 337-341.

Oscáriz, J. C., \& Pisabarro, A. G. (2010). Characterization and mechanism of action of cerein 7, a bacteriocin produced by Bacillus cereus Bc7. Journal of applied microbiology, 89(2), 361-369.

P A, R., P, R., I K, H., \& A-B, K. (2010). Characterization of a broad range antimicrobial substance from Bacillus cereus. Journal of applied microbiology, 96(4), 648-655.

Sharafi, H., Abdoli, M., Hajfarajollah, H., Samie, N., Alidoust, L., Abbasi, H., et al. (2014). First Report of a Lipopeptide Biosurfactant from Thermophilic Bacterium Aneurinibacillus thermoaerophilus MK01 Newly Isolated from Municipal Landfill Site. Applied Biochemistry and Biotechnology, 173(5), 1236-1249.

Thakur, Yaday, Gogoi, BK, Bora, \& TC (2007). Isolation and screening of Streptomyces in soil of protected forest areas from the states of Assam and Tripura, India, for antimicribial metabolites. Journal de Mycologie Medicale, 17, 242-246. 
Wang, B., \& Fang, R. (2011). Research Progress on Selenium-enriched Probiotics of Nutrition Function. Feed Review, (5), 36-38.

Xiang, Y., Chen, Z., Luo, C., Zhou, H., \& Liu, Y. (2015). The Antifungal Activities of Bacillus spp. and Its Relationship with Lipopeptide Antibiotics Produced by Bacillus spp. Scientia Agricultura Sinica, (20), 4064-4076.

Xiang, Y., Zhou, H., Liu, Y., \& Chen, Z. (2016). Isolation and Identification of Lipopeptide Antibiotics Produced by Bacillus amyloliquefaciens B1619 and the Inhibition of the Lipopeptide Antibiotics to Fusarium oxysporum f.sp.lycopersici. Scientia Agricultura Sinica, (15), 2935-2944.

Yakimov, M. M., Timmis, K., Wray, V., \& Fredrickson, H. L. (1995). Characterization of a new lipopeptide surfactant produced by thermotolerant and halotolerant subsurface Bacillus licheniformis BAS50. Applied and Environmental Microbiology, 61(5), 1706-1713.

Zhang, L. (2012) Identification of A Bacillus cereus and Isolation and Study on Its Antimicrobial Substance. South China University of Technology

Zhang, Z., Zhao, Q., Yu, J., Liu, C., Xu, H., \& Gu, W. (2019). Selection of Culture Medium and Optimization of Fermention Conditions for Bacillus subtilis Producing Antimicrobial Peptides. China Animal Husbandry \& Veterinary Medicine, 46(4), 269-278.

\section{Figures}

\section{Figure 1}

Comparison of antibacterial activity of different antimicrobial substance produced by Bacillus cereus $₫ \mathrm{~A}$, antibacterial substance obtained by acid precipitation method. B, antibacterial substance obtained by ammonium sulfate precipitation method. $C$, the supernatant containing antibacterial substance. $D$, the blank control $\bigotimes$ 


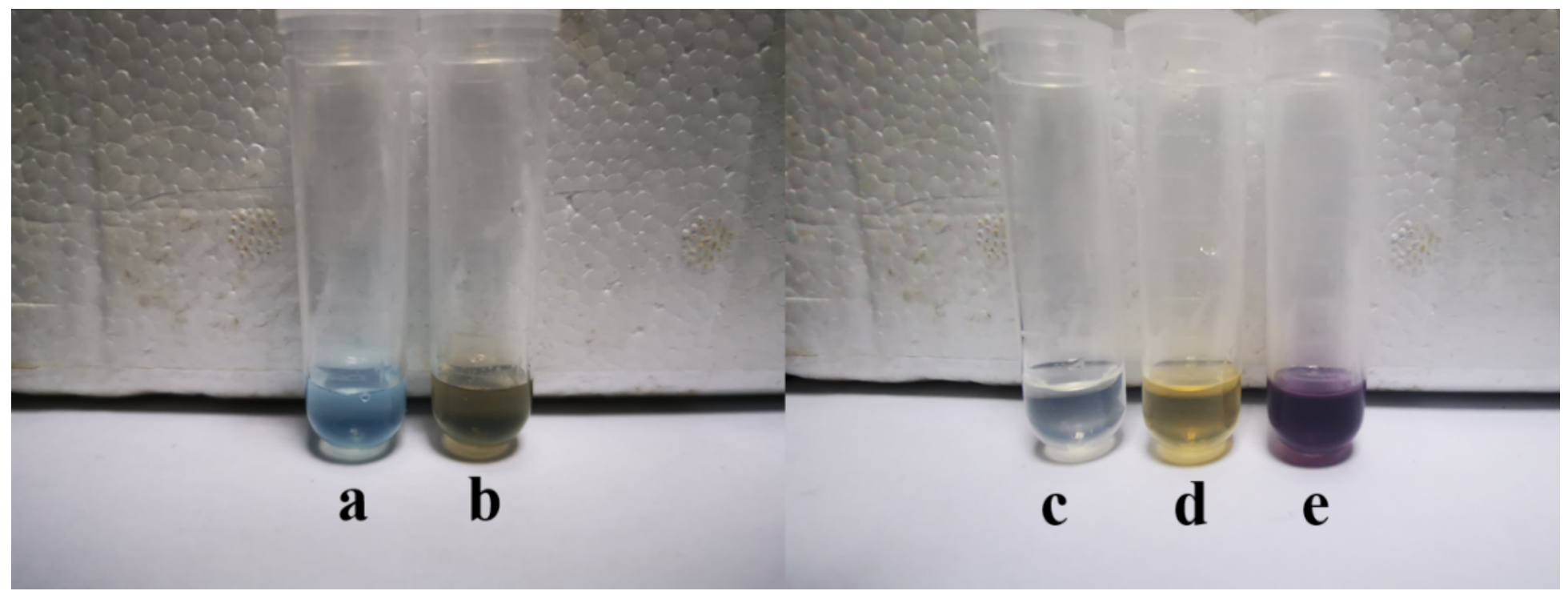

\section{Figure 2}

Biuret reaction $(a, b)$ and ninhydrin reaction $(c, d, e)$ of antibacterial substance, in which a and $c$ are blank control of pure water, $b$ is biuret reaction of antibacterial substance, $d$ is solution of antibacterial substance added with ninhydrin, and $\mathrm{e}$ is the antibacterial substance solution boiled after adding ninhydrin. 


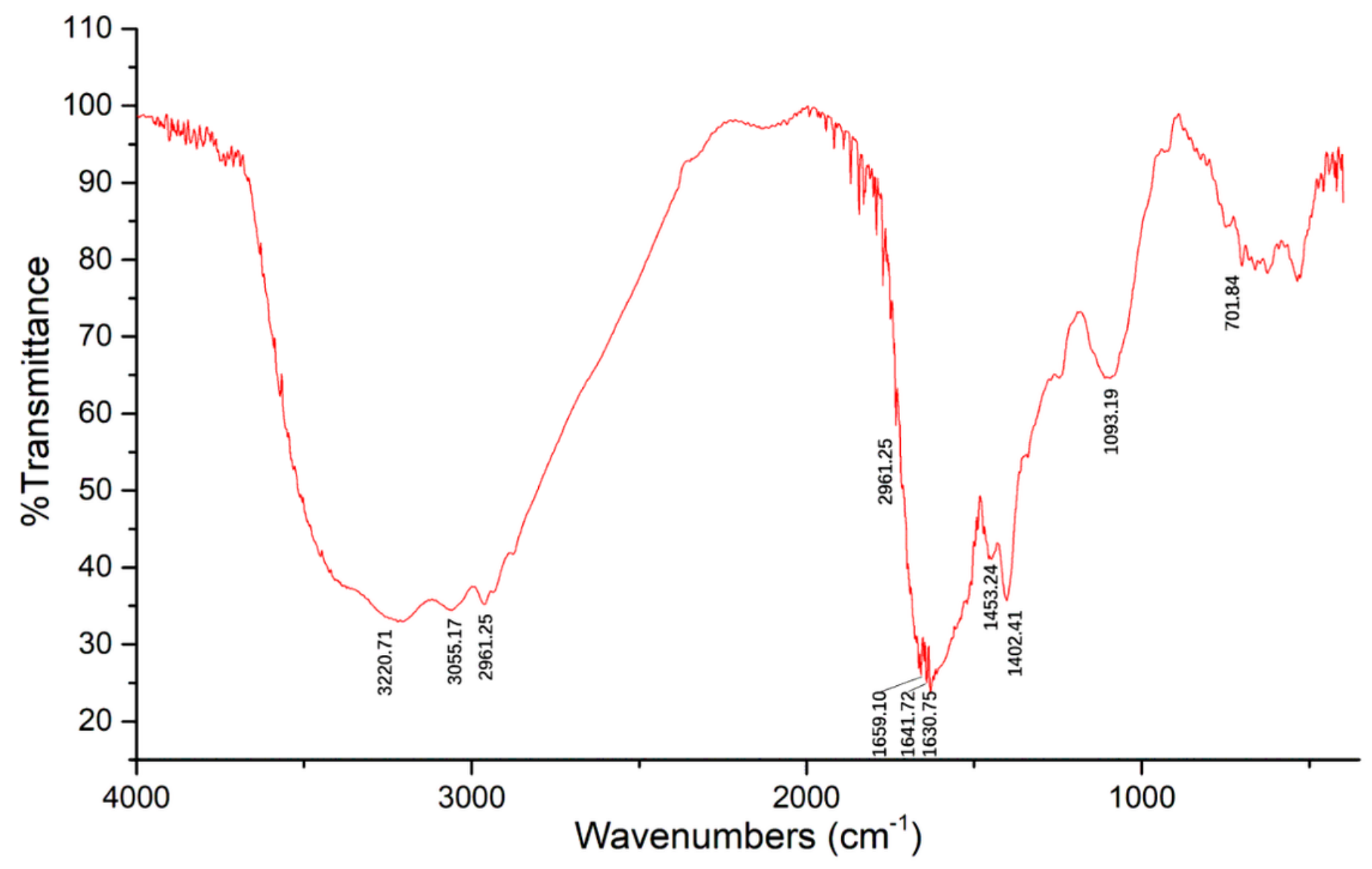

Figure 3

The IR spectra of antimicrobial substance from Bacillus cereus.

A

1008.502

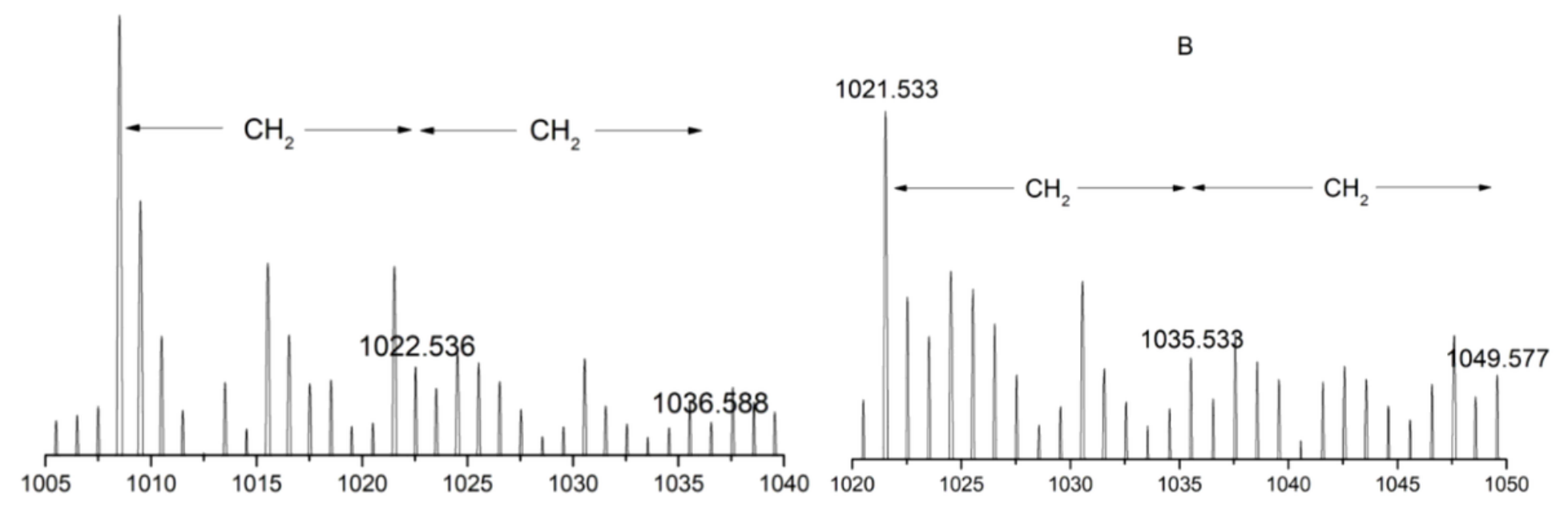

Figure 4 
MALDI-TOF-MS of antimicrobial substance produced by Bacillus cereus.

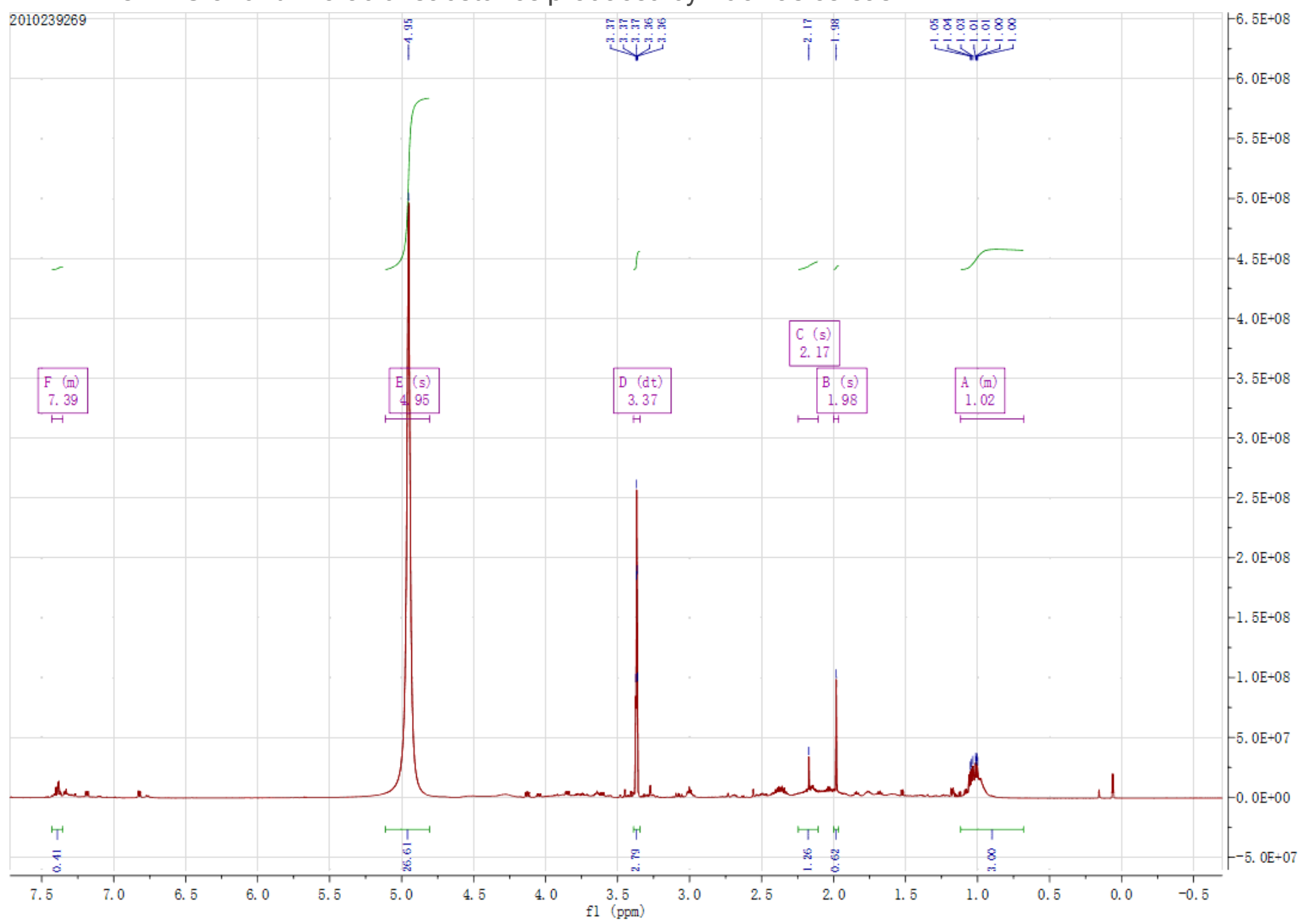

Figure 5

1H NMR spectra of antibacterial substance produced by Bacillus cereus.

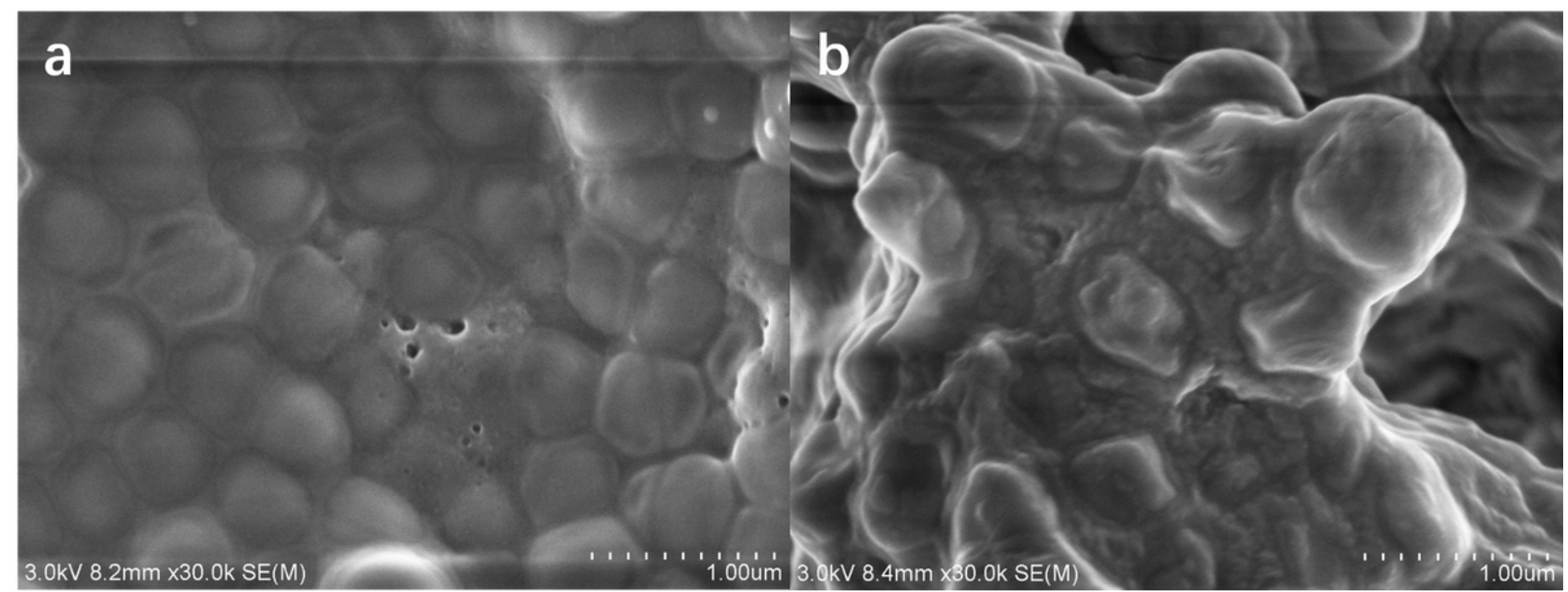

Figure 6 
Effect of antimicrobial substance produced by Bacillus cereus on cell morphology and structure of Staphylococcus aureus.
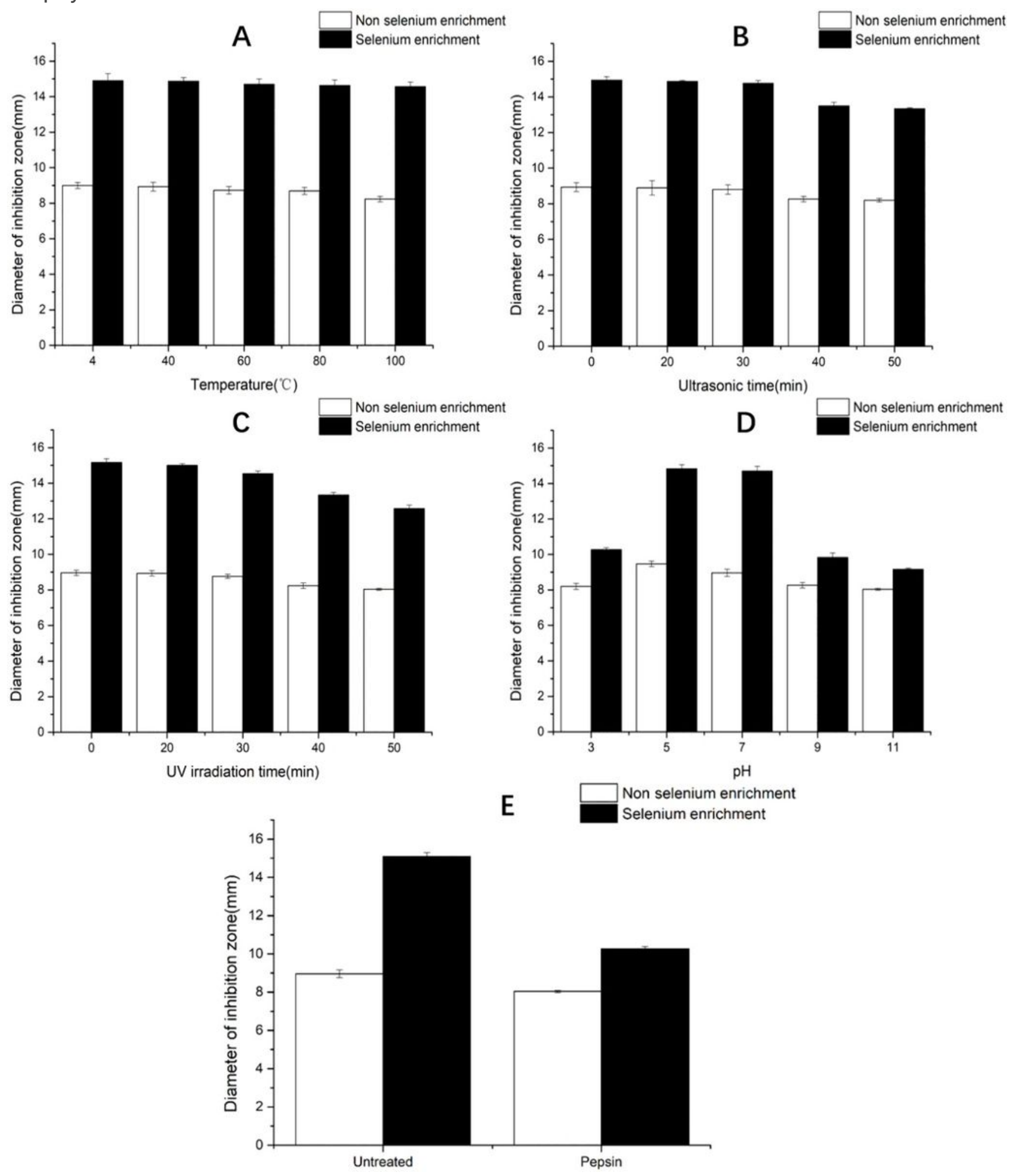

Figure 7

Stability analysis of antibacterial substance produced by Bacillus cereus against temperature(A), ultrasonic time(B), UV(C), acid-base(D) and pepsin(E). 